\title{
Empfehlungen der AWMF zum Umgang mit Interessenkonflikten bei Fachgesellschaften
}

\section{Zusammenfassung}

Die bei der Delegiertenkonferenz im Mai 2009 eingesetzte ad-hocKommission der AWMF hat Empfehlungen zum Umgang mit Interessenkonflikten bei Fachgesellschaften erarbeitet, die das Präsidium der AWMF am 23. April 2010 bei seiner Sitzung in Frankfurt/Main verabschiedet hat. Der volle Wortlaut der Empfehlungen mit Literaturangaben wird hier publiziert:

\section{Text}

\section{Empfehlungen der AWMF zum Umgang mit Interessenkonflikten bei Fachgesellschaften}

Erarbeitet von einer ad-hoc-Kommission der AWMF* und verabschiedet vom Präsidium der AWMF am 23. April 2010

*Mitglieder: Prof. Dr. med. Hartwig Bauer, Dr. med. Manfred Gogol, Prof. Dr. med. Toni Graf-Baumann, Prof. Dr. med. Axel Haverich, Prof. Dr. med. David Klemperer, Prof. Dr. rer. biol. hum. Dipl.-Math. Hans-Konrad Selbmann, Prof. Dr. med. Claudia Spies, Prof. Dr. med. Peter von Wichert, Dr. jur. Albrecht Wienke

Die Bedeutung von Interessenkonflikten und die Notwendigkeit, diese offen darzulegen und bei Entscheidungen zu berücksichtigen, sind zunehmend in den Fokus öffentlicher Diskussion gerückt [1],[2]. Die Relevanz von Interessenkonflikten bei medizinisch-wissenschaftlichen Fachgesellschaften ergibt sich durch ihre Aktivitäten bei Publikationen von Studien, in der Fort- und Weiterbildung sowie bei der Leitlinienentwicklung. Medizinisch-wissenschaftliche Fachgesellschaften sind aufgefordert - wie andere Institutionen, die sich an Patientenversorgung, Forschung und Lehre, Fort- und Weiterbildung sowie an der Erstellung von Leitlinien beteiligen, Verfahren zum Umgang mit Interessenkonfliktverfahren einzuführen und anzuwenden. Die AWMF hat deshalb Empfehlungen zum Umgang mit Interessenkonflikten für Publikationen in Fachgesellschaftsorganen, für Fachgesellschaftskongresse und Leitlinienentwicklung erarbeitet.

"Interessenkonflikte sind definiert als Gegebenheiten, die ein Risiko dafür schaffen, dass professionelles Urteilsvermögen oder Handeln, welches sich auf ein primäres Interesse beziehen, durch ein sekundäres Interesse unangemessen beeinflusst werden." "Conflicts of interest are defined as circumstances that create a risk that professional judgments or actions regarding a primary interest will be unduly influenced by a secondary interest." [[3] S. 6] Diese Definition liegt dem Report des Institute of Medicine über Interessenkonflikte zugrunde [IOM; [3]. Es handelt sich um eine Weiterentwicklung der Definition von Thompson[4], [5] . Die Definition enthält vier wesentliche Elemente: professionelles Urteilsvermögen, primäres und sekundäres Interesse sowie unangemessene Beeinflussung [6], [7], [8]. Dabei ist ein Interessenkonflikt ein Zustand und nicht - wie häufig angenommen - das Ergebnis einer Handlung oder ein verzerrtes Urteil oder eine verzerrte Bewertung. Ein Interessenkonflikt kann materieller, psychologischer oder sozialer Art sein und hat nichts damit zu tun, ob sich eine Person beeinflusst fühlt oder nicht [9], [10], [11], [12], [13], [14], [15], [16], [17].

\section{Empfehlungen zur Offenlegung von Interessenkonflikten im Interesse von Fachgesellschaften}

Das Ziel von Regelungen zu Interessenkonflikten ist es, die Integrität der professionellen Urteilsfähigkeit zu schützen und das Vertrauen der Öffentlichkeit zu bewahren [3].

Interessenkonflikte sind nach vier grundsätzlichen Prinzipien offenzulegen [18], [19], [20]:

- Trennungsprinzip: Entgeltliche oder unentgeltliche Zuwendungen müssen unabhängig von Entscheidungen bzw. Geschäften sein.

- Transparenzprinzip: Jede entgeltliche oder unentgeltliche Zuwendung muss offengelegt werden. Alle Leistungen an eine medizinische Einrichtung, an einen Arzt oder an einen anderen Mitarbeiter im Gesundheitswesen müssen dem Arbeitgeber mitgeteilt, schriftlich fixiert und genehmigt werden.

- Äquivalenzprinzip: Leistung und Gegenleistung müssen in einem angemessenen Verhältnis stehen.

- Dokumentationsprinzip: Alle Leistungen müssen schriftlich festgehalten werden. In den schriftlichen Vereinbarungen wird detailliert festgelegt, welcher Art 
etwa die Zuwendung ist, welchen Zweck sie hat und welche Leistungen konkret erbracht werden.

Die Offenlegung individueller und institutioneller Interessenkonflikte ist ein notwendiger erster Schritt [19], aber nicht hinreichend. Alle folgenden Empfehlungen beruhen auf den von Steinbrook [21] ausgewählten und modifizierten Vorschlägen des „Institute of Medicine“ (IOM).

\section{Publikationen in Fachgesellschaftsorganen}

Für die Publikationen sind international entsprechende von der IOM empfohlene Standards für Inhalt, Format und Vorgehensweisen bezüglich finanzieller Interessenkonflikte in Verbindung mit der Industrie im Rahmen einer Konsensfindung auf verschiedenen Ebenen empfohlen (IOM Empfehlung 3.3) [21], [22], [23], [24]. Als Option für Inhalt und Form der Offenlegung könnten die Kriterien des „International Committee of Medical Journal Editors“ (ICMJE) Verwendung finden [25]. Die Offenlegung von Interessenkonflikten sollte in allen Zeitschriften der Fachgesellschaften und ihren Berufsverbänden bindend sein [26], [27]. In die Bewertung der Qualität klinischer Studien sollte auch die Beurteilung der Unabhängigkeit von externer Einflussnahme eingehen.

\section{Fachgesellschaftskongresse}

Viele Fachgesellschaften stehen bei der Organisation von Fachgesellschafts-Kongressen mit der Industrie in finanziellen Verbindungen [28], [29]. Von Seiten der Industrie existieren eigene Kodizes [18], [19].

Bei jedwedem Sponsoring ist vorauszusetzen, dass eine gleichwertige Gegenleistung des Veranstalters an den Sponsor erfolgt. Mögliche Gegenleistungen zur Honorierung des Sponsors sind etwa eine Anzeigenschaltung im Kongressprogramm, Banner auf der Kongresshomepage, das Verteilen von Werbematerial, das Gewähren von Ausstellungsfläche oder Raum für das Abhalten von Satelliten-Symposien. Für die Ermittlung der Gleichwertigkeit der Leistungen sind marktübliche Preise für die Gegenleistung anzusetzen. Die Förderung einer Veranstaltung muss sich darüber hinaus auf einen objektiven und sachlichen Grund stützen, z.B. auf den wissenschaftlichen Inhalt des Programms der Veranstaltung ohne einen Bezug zu den Produkten des Sponsors. Der Ort der Veranstaltung sollte der wissenschaftlichen Zielsetzung angemessen sein, und der Anreiz zur Teilnahme sollte nicht im Freizeit- oder Erholungswert des Tagungsorts liegen. Das wissenschaftliche Programm der Veranstaltung sollte im Vordergrund stehen ( $\S 8$ Abs. 1 Kodex Medizinprodukte) [18], Freizeit- und Unterhaltungselemente, insbesondere ein Rahmenprogramm mit Ausflügen, Show-Entertainment, kostenlose Abgabe von Festabendkarten an Kunden und VIP Limousinenser- vice durch die Industrie sind unzulässig und dürfen auch nicht von Industrieunternehmen außerhalb des Veranstaltungsprogramms organisiert werden. Die Bewirtung von Teilnehmern bei Kongressen sollte nur im erforderlichen Umfang erfolgen, um die Teilnehmer zu stärken, damit sie den fachlichen Inhalten der Veranstaltung weiter folgen können (z.B. gemeinsames Mittagessen, Pausenbewirtung mit Gebäck oder belegten Brötchen, Fingerfood, Heiß- und Kaltgetränke). Zulässig sind nach der Rechtsprechung Arbeitsessen mit Kunden am Rande von Kongressen, wenn sie der Planung und Organisation gemeinsamer Projekte dienen (BGH NStZ-RR 2003, S. 171 f.) und einen sozial üblichen Rahmen nicht überschreiten. Unproblematisch ist ebenfalls die sozialadäquate Bewirtung am Kongress-Werbestand, die grundsätzlich jedem interessierten Teilnehmer angeboten wird. Sponsorenverträge sind mit der Fachgesellschaft, die Veranstalter des Kongresses ist, und ihren beauftragten Agenturen abzuschließen (zuletzt BGH NJW 2008, S. 3580 - EnBW) [27], [30], [31], [32].

Bei der Anmeldung der Veranstaltungen bei den Ärztekammern zur Vergabe von CME Punkten soll entsprechend den oben genannten Prinzipien vorgegangen werden (Empfehlungen der Bundesärztekammer zur ärztlichen Fortbildung vor dem Hintergrund des $\S 95$ d SGB V) [33]. Von jedem einzelnen Referenten ist durch die Fachgesellschaften einzufordern, dass jeder z.B. entsprechend des ICMJE Standards seine Interessenkonflikte vor Beginn der Veranstaltung offen legt [25]. Es muss ferner sichergestellt werden, dass die Fortbildungsangebote an den Lernbedürfnissen und Lernnotwendigkeiten der Ärzte ansetzen. Dabei könnten Umfragen innerhalb der Fachgesellschaften bei ihren Mitgliedern wichtig sein, um diese Bedürfnisse und Notwendigkeiten zu erfragen (DIN EN ISO 9001:2008 Standard).

Fachgesellschaften sollten ihre Kongresse einschließlich des Finanzierungssystems so gestalten (IOM Empfehlung 5.3) [21], dass sie von der Industrie weitestgehend unbeeinflusst sind, das öffentliche Vertrauen in die Integrität der Fachgesellschaften verstärken und ein Fort- und Weiterbildungsprogramm von hoher Qualität anbieten. Falls eine Finanzierung über die Industrie erfolgt, soll diese detailliert und standardisiert offengelegt werden (z.B. durch Angaben des Sponsorings im Kongressprogramm). Es obliegt den Landesärztekammern, die Kriterien für die Vergabe von CME-Punkten für diese Veranstaltungen - bei turnusmäßigen Veranstaltungen unter Berücksichtigung der Evaluation durch Teilnehmer mit gezielten Fragen zur Wahrnehmung eines Sponsoreneinflusses bzw. durch stichprobenartige Audits - festzulegen. 


\section{Leitlinienentwicklung}

An der Leitlinienentwicklung wirken mandatierte Vertreter von Fachgesellschaften und anderen Organisationen mit. Die Basisstruktur jedes Leitlinien-Entwicklungsvorhabens bilden das Lenkungsgremium, die Koordinatoren und das Konsentierungsgremium. Bei kleineren Leitlinien-Gruppen kann das Lenkungsgremium dem Konsentierungsgremium entsprechen. Das Lenkungsgremium legt gemeinsam mit den Koordinatoren die Autoren der Leitlinie fest. Neben den genannten Personenkreisen kann es externe Experten geben, die zum Wissen der Leitlinie beitragen, ohne dass sie an der Bewertung der Evidenzen und der Konsensfindung beteiligt sind.

Zu Beginn der Leitlinienarbeit sollen das Lenkungsgremium und die Koordinatoren namentlich bekannt sein. Die Benennung der Mitglieder des Konsentierungsgremiums, von Arbeitsgruppen und Autoren kann bedarfsbezogen im Verlauf des Entwicklungsfortschrittes erfolgen.

Das AWMF-Regelwerk sieht folgendes Vorgehen vor (www.awmf-leitlinien.de):

1. Das Finanzierungskonzept einer Leitlinienentwicklung muss bei deren Anmeldung bei der AWMF durch die federführenden Fachgesellschaften offengelegt werden. Eine Finanzierung durch Dritte mit direkter Einflussnahme führt zur Ablehnung der Anmeldung [34], [35], so wie dies international auch empfohlen und praktiziert wird [27], [36], [37], [38], [39]. Interessenkonflikte werden grundsätzlich schriftlich mit Hilfe eines Formblattes erklärt (online unter: www.le itlinien.net/II-tools/II-konflikt.pdf), das materielle und immaterielle Interessen umfasst. Zu den immateriellen Interessen gehören ggf. die mandatierende Organisation (z.B. Fachgesellschaft), der Arbeitgeber und der wissenschaftliche Schwerpunkt der betroffenen Person.

2. Die Erklärungen der Interessenkonflikte der Mitglieder des Lenkungsgremiums, die Koordinatoren und die Leiter der Arbeitsgruppen müssen zu Beginn der Leitlinienarbeit vorliegen. Es ist Aufgabe der Koordinatoren, die Interessenkonflikterklärungen der später hinzutretenden Mitglieder der Arbeitsgruppen und des Konsentierungsgremiums sowie anderer einzufordern und zusammenzutragen.

3. Die Interessenkonflikterklärungen der Mitglieder des Lenkungsgremiums werden von den Präsidien der sie entsendenden Fachgesellschaften zur Kenntnis genommen und bezüglich ihrer Befangenheit bewertet. Die Erklärungen der Interessenkonflikte aller anderen Mitwir-kenden werden vom Lenkungsgremium und den Koordinatoren bewertet. Es ist beabsichtigt, zusammen mit der AWMF-Leitlinienkommission eine
Befangenheitsskala zu entwickeln, um eine Reproduzierbarkeit der Bewertungen zu gewährleisten.

4. Mitwirkende mit Interessenkonflikten, die aufgrund der Fachgesellschaften bzw. anderer Organisationen oder durch das Lenkungsgremien als befangen bewertet wurden, sollen nicht an der Bewertung der Evidenzen und der Konsensfindung mitwirken (Empfehlung IOM 4.1) [21], [36], [37], [38]. Sie haben, sofern auf inr Wissen nicht verzichtet werden kann, den Status von externen Experten. Bei den Autoren ist besonders auf die Geringfügigkeit der Interessenkonflikte zu achten [34].

5. Die Interessenkonflikterklärungen aller Mitwirkenden sind im Leitlinienreport der Leitlinie im Detail wieder zugeben. Die Langfassung der Leitlinie muss das Verfahren der Erfassung und der Bewertung von Interessenkonflikten mit Verweis auf den Leitlinienreport beschreiben.

6. Fertige Leitlinien, bei denen die Finanzierung Interessenkonflikte enthält oder die Interessenkonflikte einzelner Mitwirkender nicht transparent sind, werden nicht in das AWMF-Register aufgenommen. Den Tatbestand prüfen die Leiter der AWMF-Leitlinienkommission, in strittigen Fällen das AWMF-Präsidium.

Das Prozedere der Erfassung und des Umgangs mit Interessenkonflikten ist Bestandteil des AWMF-Regelwerks, das von der Leitlinienkommission der AWMF erarbeitet, umgesetzt und fortgeschrieben wird.

Zusammenfassend ist zu betonen, dass zum einen die Zusammenarbeit der Fachgesellschaften mit der Industrie eine wichtige Voraussetzung für die Entwicklung innovativer Therapiekonzepte zum Wohle des Patienten ist, zum anderen diese Interaktion durch Interessenkonflikte in unterschiedlichem Ausmaß beeinträchtigt sein kann. Die von den Fachgesellschaften erarbeiteten und konsentierten Regelungen sollen dazu beitragen, neben dem professionellen Eigenanspruch den gesetzlichen Vorgaben zu genügen und so für alle Beteiligten im Sinne des Wohles unserer Patienten eine bestmögliche Sicherheit zu schaffen.

\section{Literatur}

1. DeAngelis CD, Fontanarosa PB. Conflicts Over Conflicts of Interest. JAMA March 20, 2009 Mit Vermerk auf Retraktion: Hopkins Tanne J. JAMA told to change ist policy on investigating competing interests. BMJ 339: b2936; 25 July 2009

2. D'Arcy E, Moynihan R. Can the relationship between doctors and drug companies ever be a healthy one? PLoS Med. 2009 Jul 21;6(7):e1000075

3. Lo B, Marilyn J. Field, Committee on Conflict of Interest in Medical Research. Conflict of Interest in Medical Research, Education, and Practice. Institute of Medicine. 2009 http://books.nap.edu/ openbook.php?isbn $=030913188 \times$ \&page $=6$

4. Thompson DF. The Challenge of Conflict of Interest in Medicine. Z Evid Fortbild Qual Gesundhwes 2009;103(3):136-140. 
5. Thompson DF. Understanding Financial Conflicts of Interest. N Engl J Med 1993;329(8):573-576.

6. Weltärztebund. Deklaration von Genf. http:// www.bundesaerztekammer.de/downloads/Genf.pdf (Zugriff 01.09.2009).

7. Bundesärztekammer. (Muster-)Berufsordnung für die deutschen Ärztinnen und Ärzte. http://www.bundesaerztekammer.de/ downloads/MBOStand20061124.pdf (Zugriff 01.09.2009).

8. Klemperer D. Interessenkonflikte: Gefahr für das ärztliche Urteilsvermögen. DÄB 2008; 105 (4): 2098-2100

9. The PLoS Medicine Editors. Making Sense of Non-Financial Competing Interests. PLoS Med 2008;5(9):e199.

10. Moynihan R. Who pays for the pizza? Redefining the relationships between doctors and drug companies. 1: Entanglement. BMJ 2003;326(7400):1189-1192.

11. Fugh-Berman A., Ahari S. Following the Script. How Drug Reps Make Friends and Influence Doctors. PLoS Med 2007;4: e150.

12. Brennan TA, Rothman DJ, Blank L, Blumenthal D, Chimonas SC, Cohen JJ, Goldman J, Kassirer JP, Kimball H, Naughton J, Smelser $\mathrm{N}$. Health industry practices that create conflicts of interest. A policy proposal for academic medical centers. JAMA 2006;295:429-433.

13. Cialdini R: Influence. The Psychology of Persuasion. Revised Ed. New York: Collins 2007.

14. Sagarin BJ, Cialdini, RB, Rice WE, Serna SB. Dispelling the illusion of invulnerability. The motivations and mechanisms of resistance to persuasion. Journal of Personality and Social Psychology. 2002; 83: 526-541.

15. Schneider N. Interessenkonflikte. Chemotherapie Journal; in press 2010

16. Dana J, Loewenstein G. A Social Science Perspective on Gifts to Physicians From Industry. JAMA 2003; 290: 252-255.

17. Grande D, Frosch DL, Perkins AW, Kahn BE. Effect of Exposure to Small Pharmaceutical Promotional Items on Treatment Preferences. Arch Intern Med 2009;169(9):887-893.

18. Kodex "Medizinprodukte". http://www.vdek.com/presse/ Broschueren/medizinkodex/medizinkodex.pdf (Zugriff 01.09.2009)

19. "Freiwillige Selbstkontrolle für die Arzneimittelindustrie e.V.". http://www.vfa.de/de/vfa/fs-arzneimittelindustrie/ fsam-dokumente/fsa-kodex.html

20. Gemeinsamer Standpunkt zur strafrechtlichen Bewertung der Zusammenarbeit zwischen Industrie, medizi-nischen Einrichtungen und deren Mitarbeitern. http:// www.medtech-kompass.de/service/index.php (Zugriff 01.09.2009).

21. Steinbrook R. Controlling conflict of interest - proposals from the Institute of Medicine. N Engl J Med 2009; 360: 2160-2163.

22. Department of Health and Human Services. Office of Inspector General. The Food and Drug Administration s oversight of clinical investigators' financial information. OEI-05-07-00730. 2009 http://www.oig.hhs.gov/oei/reports/oei-05-07-00730.pdf.

23. Scott-Lichter $D$ and the Editorial Policy Committee, Council of Science Editors. CSE's White Paper on promoting integrity in scientific journal publications, 2009 update. http:// www.councilscienceeditors.org/services/policies.cfm.

24. Blum JA, Freeman K, Dart RC, Cooper RJ. Requirements and definitions in conflict of ineterst policies of medical journals. JAMA 2009; 302 (20): 2230-2234
25. Drazen JM, Van Der Weyden MB, Sahni P, Rosenberg J, Marusic A, Laine C, Kotzin S, Horton R, Hébert PC, Haug C, Godlee F, Frizelle FA, de Leeuw PW, Deangelis CD. Uniform Format for Disclosure of Competing Interests in ICMJE Journals. N Engl J Med. 2009 Nov 5;361(19):1896-7. Epub 2009 Oct 13

26. Schneider N, Lingner H, Schwartz FW. Disclosing conflicts of interest in German publications concerning health services research. BMC Health Services Research 2007; 7:78

27. www.eanm.org. European Association of Nuclear Medicine (EANM) Guiding principles with regard to transparency, version 04.11.2009.

28. Robertson J, Moynihan R, Walkom E, Bero L, Henry D. Mandatory disclosure of pharmaceutical industry-funded events for health professionals. PLoS Med 2009;6:e1000128.

29. Okike K, Kocher MS, Wei EX, MehIman CT, Bhandari M. Accuracy of conflict-of-interest disclosures reported by physicians. N Engl J Med. 2009 Oct 8;361(15):1466-74.

30. Wagner J, Moosburner R. Rechtliche Gestaltung von Kooperationen mit der Industrie bei medizinischen Fachkongressen. Mitteilungen der Deutschen Gesellschaft für Chirurgie 2009; 38:255-256

31. Schneider N, Lückmann SL. Pharmasponsoring in der ärztlichen Fort- und Weiterbildung. Z Allg Med 2008; 84:516-524.

32. Treede RD, May A. Kodex für den Deutschen Schmerzkongress. Vereinbarung der DGSS und DMKG. http://www.dmkg.de/pdf/ kodex_schmerzkongress2009.pdf, 19.03.2009.

33. Wienke A. Die neue ärztliche Fortbildung - Das Ende des PharmaSponsorings? Hessisches Ärzteblatt 2005, 3:177-178. http:// www.laekh.de/upload/Hess._Aerzteblatt/2005/2005_03/2005_ 03_11.pdf

34. Spies C, Kopp I, Krämer M, Kastrup M, Braun J, Taeger K, Schüttler J. Leitlinien und deren Relevanz in klinischen Behandlungspfaden - Grundlagen und Stellenwert in der interdisziplinären Zusammenarbeit. Anästh Intensivmed 2006; 47:675-683.

35. Eggers V, Kopp I, Spies C. Do we need a reform in the development of guidelines? A commentary. Anaesthesist. 2009 Jul; 58(7):728-30.

36. Sniderman $A D$, Furberg $C D$. Why guideline-making requires reform. JAMA 2009; 301:429-431.

37. Choudhry NK, Stelfox HT, Detsky AS. Relationships between authors of clinical practice guidelines and the pharmaceutical industry. JAMA 2002; 287:612-617.

38. Holloway RG, Mooney CJ, Getchius TSD, Edlund WS, Miyasaki J. Invited article: Conflicts of interest for authors of American Academy of Neurology clinical practice guidelines. Neurology 2008; 71:57-63.

39. Schünemann HJ, Osborne M, Moss J, Manthous C, Wagner G, Sicilian L, Ohar J, McDermott S, Lucas L, Jaeschke R; ATS Ethics and Conflict of Interest Committee and the Documents Development and Implementation Committee. An official American Thoracic Society Policy statement: managing conflict of interest in professional societies. Am J Respir Crit Care Med. 2009 Sep 15; 180(6): 564-80.

40. Rothmann DJ, McDonald WJ, Berkowitz CD, Chimonas SC, DeAngelis CD, Hale RW, Nissen SE, Osborn JE, Scully Jr JH, Thomson GE, Wofsy E. Professional medical associations and their relationships with industry. A proposal for controlling conflict of interest. JAMA 2009;301:1367-1372. 
Korrespondenzadresse:

M.A. Wolfgang Müller

AWMF-Geschäftsstelle, Ubierstr. 20, 40223 Düsseldorf office@awmf.org

\section{Bitte zitieren als}

Müller W. Empfehlungen der AWMF zum Umgang mit

Interessenkonflikten bei Fachgesellschaften. GMS Mitt AWMF.

2010; 7:Doc8.

DOI: 10.3205/awmf000206, URN: urn:nbn:de:0183-awmf0002063
Artikel online frei zugänglich unter

http://www.egms.de/en/journals/awmf/2010-7/awmf000206.shtml

Eingereicht: 23.04.2010

Veröffentlicht: 28.04 .2010

\section{Copyright}

(C2010 Müller. Dieser Artikel ist ein Open Access-Artikel und steht unter den Creative Commons Lizenzbedingungen

(http://creativecommons.org/licenses/by-nc-nd/3.0/deed.de). Er darf vervielfältigt, verbreitet und öffentlich zugänglich gemacht werden, vorausgesetzt dass Autor und Quelle genannt werden. 